\title{
ON THE GLEASON AND HARNACK METRICS FOR UNIFORM ALGEBRAS
}

\section{HEINZ KÖNIG}

Let $B(X, R)$ and $B(X, C)$ consist of the bounded real and complex valued functions on the set $X$ with $\|\cdot\|$ the supnorm on $X$. For $1 \in A$ $\subset B(X, C)$ a complex subalgebra which separates the points of $X$ one defines

$G: G(u, v)=\operatorname{Sup}\{|f(u)-f(v)|: f \in A$ with $\|f\| \leqq 1\} \quad$ for $u, v \in X$, $\sigma: \sigma(u, v)=\operatorname{Sup}\{|f(v)|: f \in A$ with $\|f\| \leqq 1$ and $f(u)=0\}$

for $u, v \in X$.

Then $G \leqq 2$ is a metric on $X$ named after Gleason [4]. For $1 \in B$ $C B(X, R)$ a real linear subspace which separates the points of $X$ one defines

$$
\begin{aligned}
H: H(u, v)= & \log \inf \left\{1 \leqq \lambda \in R \text { with }\left(^{*}\right): F(u) \leqq \lambda F(v)\right. \\
& \text { and } F(v) \leqq \lambda F(u) \text { for all } 0 \leqq F \in B\} \quad \text { for } u, v \in X,
\end{aligned}
$$

where $H(u, v)=\infty$ if no $1 \leqq \lambda \in R$ with $\left(^{*}\right)$ exists. Then $H \leqq \infty$ is a metric (in the extended sense) on $X$ named after Harnack [1]. It admits the beautiful interpretation as the intrinsic metric for the state space $S(B)=\left\{\phi \in B^{*}: \quad \phi(1)=\|\phi\|=1\right\} \subset B^{*}$ restricted to $X \subset S(B)$ [2]. In the case $B=\operatorname{Re} A$ one has the famous result that $G(u, v)<2 \Leftrightarrow \sigma(u, v)<1 \Leftrightarrow H(u, v)<\infty$ for $u, v \in X[3],[1]$. This is an equivalence relation on $X$ which produces the Gleason parts for $A$. Furthermore $G$ and $H$ are equivalent metrics on $X[1]$. We claim the subsequent quantitative version of these facts. An essential part of the proof is contained in [5].

Theorem. In the case $B=\operatorname{Re} A$ we have

$$
H(u, v)=\log \frac{1+\sigma(u, v)}{1-\sigma(u, v)}=2 \log \frac{2+G(u, v)}{2-G(u, v)} \quad \text { for } u, v \in X .
$$

Proof. We can assume $A$ to be norm closed. Let $u, v \in X$. From [5, Satz 1.8] we have $\sigma(u, v)=4 G(u, v) /\left(4+(G(u, v))^{2}\right)$ or $(1-\sigma(u, v)) /(1+\sigma(u, v))=\left(\left(2-G\left(\begin{array}{ll}u, v & )\end{array}\right) /(2+G(u, v))\right)^{2}\right.$. Thus $\sigma(u, v)<1 \Leftrightarrow G(u, v)<2$. And from [5, Spezialfall 1.5] we have

$$
((1-\sigma(u, v)) /(1+\sigma(u, v))) F(u) \leqq F(v)
$$

Received by the editors October 18, 1968. 
for all $0 \leqq F \in \operatorname{Re} A$. Thus if $\sigma(u, v)<1$, then

$$
\lambda=(1+\sigma(u, v)) /(1-\sigma(u, v))
$$

fulfills $\left({ }^{*}\right)$. For the converse we claim that if $1 \leqq \lambda \in R$ fulfills $\left.{ }^{*}\right)$ then $\sigma(u, v) \leqq(\lambda-1) /(\lambda+1)$. It follows that $\sigma(u, v)<1$ and $\lambda \geqq(1+\sigma(u, v)) /(1-\sigma(u, v))$ so that the assertation will be proved. We take $f \in A$ with $\|f\| \leqq 1$ and $f(u)=0$ and have to show that $|f(v)| \leqq(\lambda-1) /(\lambda+1)$. We can assume that $\|f\|<1$ and $f(v) \geqq 0$. Then $P=(1+f) /(1-f) \in A$ with $\operatorname{Re} P=\left(1-|f|^{2}\right) /|1-f|^{2} \geqq 0$. Thus

$$
\operatorname{Re} P(v)=(1+f(v)) /(1-f(v)) \leqq \lambda \operatorname{Re} P(u)=\lambda
$$

or

$$
f(v) \leqq(\lambda-1) /(\lambda+1) .
$$

The proof is complete.

Remark Added in Proof: Because of [5, Korollar 1.3 and Korollar 1.6] the function $\sigma$ is also a metric on $X$ which in view of the above theorem is equivalent to $H$ and $G$. The author is indebted to the referee for this remark.

\section{REFERENCES}

1. H. S. Bear, A geometric characterization of Gleason parts, Proc. Amer. Math. Soc. 16 (1965), 407-412.

2. H. S. Bear and Max L. Weiss, An intrinsic metric for parts, Proc. Amer. Math. Soc. 18 (1967), 812-817.

3. Errett Bishop, Representing measures for points in a uniform algebra, Bull. Amer. Math. Soc. 70 (1964), 121-122.

4. A. M. Gleason, Function algebras, Seminar on analytic functions, Vol. 2, Institute for Advanced Study, Princeton, N. J., 1957.

5. Heinz König, Zur abstrakten Theorie der analytischen Funktionen. II, Math. Ann. 163 (1966), 9-17.

Universität des SaARlandes, SAarbrücken, Germany 